Research Article

\title{
Detection Performance Analysis of the Standard FDA and FDA-MIMO Radar in the Gaussian Background
}

\author{
Yongze Liu $\mathbb{D},{ }^{1}$ Yuehong $M a\left(\mathbb{D},{ }^{2}\right.$ and Haiming Jing $\mathbb{D}^{1}$ \\ ${ }^{1}$ School of Information Science and Technology, Shijiazhuang Tiedao University, Shijiazhuang 050043, China \\ ${ }^{2}$ School of Electrical and Electronic Engineering, Shijiazhuang Tiedao University, Shijiazhuang 050043, China \\ Correspondence should be addressed to Yongze Liu; liuyongze@stdu.edu.cn
}

Received 20 September 2021; Revised 3 November 2021; Accepted 11 November 2021; Published 25 November 2021

Academic Editor: Lorenzo Crocco

Copyright (c) 2021 Yongze Liu et al. This is an open access article distributed under the Creative Commons Attribution License, which permits unrestricted use, distribution, and reproduction in any medium, provided the original work is properly cited.

This paper studies the optimal detection performance of the standard frequency diverse array (FDA) radar and FDA multi-input multioutput (FDA-MIMO) radar in Gaussian clutter and noise. Array signal processing scheme at the receiver is firstly designed to obtain the array steering vector containing range, azimuth, and frequency increment. For the two array configurations, namely, collocated transmit-receive and collocated transmit distributed receive, the likelihood ratio test statistics and the test statistic distributions are derived in the Neyman-Pearson sense. It is then investigated how the number of array elements influences the detection performance of various radar systems at low signal-to-noise ratio (SNR). Several numerical simulations are carried out to demonstrate that the performance improvement is hard for MIMO and FDA-MIMO by only increasing the number of transmit elements, while it is achievable for the FDA. The paper finally makes a comparative analysis for detection performances of five radar configurations under different SNRs.

\section{Introduction}

It is known that frequency diverse array (FDA) radar has more promising applications in interference rejection, target detection, synthetic aperture radar (SAR) imaging, and so on [1-4]. The beam of the FDA radar can scan the space in a periodic manner by employing a tiny frequency offset across the transmit elements. Its beam pointing will change with the range, angle, time, and even frequency increment. One of the main advantages of the FDA is the flexible transmit beam control [5-9]. In the standard FDA, range and angle are coupled in the transmit beam pattern. To overcome this drawback, more researchers employ the combination of FDA and multi-input multioutput (MIMO) radar technologies for range and angle estimation, deceptive jamming suppression, and ambiguous clutter suppression $[3,10,11]$.

Fishler et al. [12] capitalized on the spatial diversity of target scattering to analyze the detection performance of statistical MIMO radar and developed an optimal detector in the Neyman-Pearson sense. Xiong et al. [13] provided theoretical performance analysis for a FDA-MIMO radar, including Cramér-Rao lower bound (CRLB), mean square error (MSE) expressions in MUSIC-based range-angle estimation algorithms, and resolution. For the problem of range ambiguity incurred by high pulse repetition frequency (PRF), Xu et al. [14] proposed a FDA-MIMO adaptive rangeangle Doppler processing approach that provides excellent performance in clutter suppression. Cui et al. [15] studied the strategies of estimating the direction of departure (DOD), direction of arrival (DOA), and range for bistatic FDA-MIMO radar. They [15] further employed nonlinear frequency increments to overcome the couple problem between the DOD and range and used the rotational invariance technique and parallel factor algorithm for parameter estimation. Lan et al. [16] devised FDA-MIMO adaptive detectors according to the generalized likelihood ratio test (GLRT) criterion, where three optimization strategies have been proposed to compute the maximum likelihood (ML) estimate of the target incremental range under the $H_{1}$ hypothesis. Lan et al. [17] further studied the problem of angle and incremental range estimation with a FDA-MIMO radar exploiting as observable a single data 
snapshot. Three estimators, CD, AMP, and AGMP-CC, are devised, where the $\mathrm{CD}$ method has a better performance. To obtain target localization in both barrage jamming and burst jamming environments, Liu et al. [18] proposed a two-step "Go Decomposition" method via alternating minimization, where a priori rank information is exploited to suppress these two kinds of jammers and extract the desired target. Zhu et al. [19] presented a unified framework detector to comparatively analyze the target detection performance of the FDA-MIMO, standard FDA, phased-array, and MIMO radar, respectively. Furthermore, the deflection coefficient and signal-to-interference-plus-noise ratio (SINR) are also adopted as performance metrics to compare their performance. The authors [19] also proved that the FDA-MIMO radar detector achieves better performance than that of the conventional radar systems.

One of the main features of the standard FDA and FDAMIMO radar is that their array steering vector contains range, azimuth angle, and frequency increment, whereas the detail of the derivation process about the array steering vector is rarely discussed in most open literature studies. Therefore, we devise the receiver architecture and present the array signal processing scheme of the standard FDA and FDA-MIMO radar in detail. Since the essential characteristics of the FDA require that the transmit array must be composed of closely spaced transmitting elements, we will study two configurations of the array, namely, collocated transmit-receive and collocated transmit distributed receive, respectively. The likelihood ratio test statistics and the test statistic distributions are derived in the Neyman-Pearson sense. The detection performances of five radar configurations are comparatively analyzed, especially in a low signal-to-noise ratio (SNR) environment.

The rest of the paper is organized as follows. In Section 2, we present the receiver architecture and signal models of the FDA and FDA-MIMO radar for the two configurations of the array structure. In Section 3, the likelihood ratio test statistics and the test statistic distributions are derived in the Neyman-Pearson sense. Section 4 presents the target detection performance of the FDA-MIMO radar, standard FDA, phased-array radar, and MIMO radar, respectively. Finally, numerical comparison results are provided in Section 5, and concluding summaries are drawn in Section 6.

\section{Signal Models}

Supposing that there are $M$ transmit elements and $N$ receive elements, the narrowband complex signal transmitted by the $m$ th element is modeled as [18]

$$
s_{m}(t)=\sqrt{\frac{E}{M}} \phi_{m}(t) e^{j 2 \pi f_{m} t},
$$

where $E$ is the total transmit energy, $f_{m}=f_{0}+m \Delta f$, $m=0,1, \ldots, M-1, f_{0}$ is the carrier frequency, $\Delta f$ is the frequency increment across the array elements, $\phi_{m}(t)$ is the transmit baseband complex envelop that satisfies the unit energy and orthogonality properties [18],

$$
\begin{gathered}
\int_{0}^{T} \phi_{l}(t) \phi_{m}^{*}(t) \mathrm{d} t=1, \\
\int_{0}^{T} \phi_{l}(t) \phi_{m}^{*}(t-\tau) e^{j 2 \pi \Delta f(m-l) t} \mathrm{~d} t= \begin{cases}0, & m \neq l, \forall \tau, \\
1, & m=l, \tau=0 .\end{cases}
\end{gathered}
$$

The received signal at the $n$th receiver is the superposition of all signals originating from various transmitters plus the additive noise. Therefore, the received signal can be expressed as $[12,18]$

$$
r_{n}(t)=\sqrt{\frac{E}{M}} \sum_{m=0}^{M-1} w_{m} \alpha_{n m}\left(f_{m}\right) \phi_{m}\left(t-\tau_{n m}\right) e^{j 2 \pi f_{m}\left(t-\tau_{n m}\right)}+n_{n}(t),
$$

where $w_{m}$ is the weight of the $m$ th transmit element, $\alpha_{n m}\left(f_{m}\right)$ is the complex-valued reflection coefficient of the target that is a function of frequency, $n_{n}(t)$ is a white, zeromean, complex Gauss random process at the $n$th receiver, and $\tau_{n m}$ is the round-trip propagation time delay and can be written as

$$
\tau_{n m}=\tau_{m}+\tau_{n}=\frac{\left(r_{t}-m d_{t} \sin \theta_{t}\right)}{c}+\frac{\left(r_{r}-n d_{r} \sin \theta_{r}\right)}{c},
$$

where $r_{t}$ and $r_{r}$ are the distance from the target to the transmit reference element and the receive reference element, respectively. $d_{t}$ and $d_{r}$ are the interspacing of transmit and receive arrays, respectively. $\theta_{t}$ is the azimuth of the line of sight of the transmit array. $\theta_{r}$ is the azimuth of the line of sight of the receive array.

Since the frequency increment $\Delta f$ and the bandwidth $B$ are small quantities compared with the carrier frequency, the reflection coefficient $\alpha_{n m}\left(f_{m}\right)$ can be considered stable and does not change with frequency. Therefore, $\alpha_{n m}\left(f_{m}\right)$ can be represented by $\alpha_{n m}$. The paper assumes that the target is composed of a finite but large number of scatterers distributed. Based on the central limit theorem, the distribution of $\alpha_{n m}$ can be approximated as complex Gauss distribution $\alpha_{n m} \sim \mathrm{CN}(0,1)[12]$.

2.1. Signal Model of the Standard FDA. For a standard FDA radar, $\phi_{m}(t)$ in (1) and $\phi_{m}\left(t-\tau_{n m}\right)$ in (4) will be omitted. To remove the effect of the time variable in the return, the schematic diagram of signal processing at the $n$th receive channel is shown in Figure 1(a) [20]. After firstly amplified by a low-noise amplifier (LNA), the signals are sent to a bandpass filter (BPF) bank, where the bandwidth of the $m$ th filter is $\min \left\{\left|\Delta f_{m}-\Delta f_{m-1}\right|\right\}$ and the central frequency is $f_{m}$. The output $r_{n m}(t)$ of the $m$ th bandpass filter is mixed with a signal of frequency $f_{m}$. At this moment, the time variable is completely removed. Therefore, the final output $r_{n}$ of the $n$th receive channel is the sum of all $r_{n m}$ and can be expressed as 


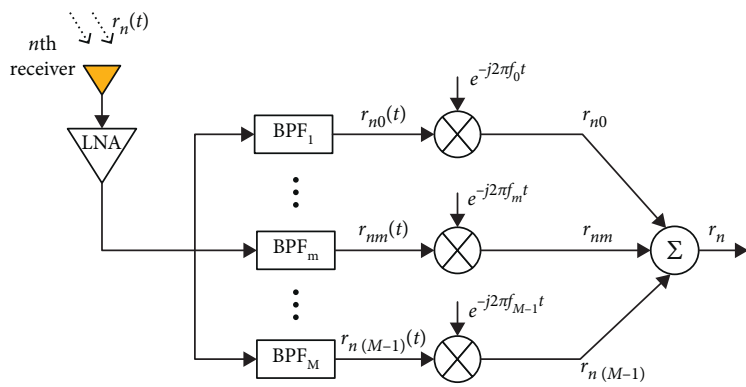

(a)

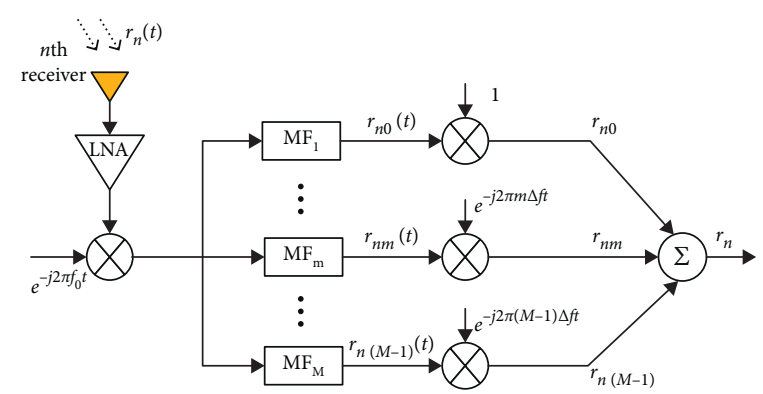

(b)

FIGURE 1: Schematic diagram of signal processing at the $n$th channel. (a) Standard FDA. (b) FDA-MIMO.

$$
r_{n}=\sqrt{\frac{E}{M}} e^{-j 2 \pi\left(\left(r_{t}+r_{r}\right) / \lambda\right)} e^{j 2 \pi\left(\left(n d_{r} \sin \theta_{r}\right) / \lambda\right)} \sum_{m=0}^{M-1} w_{m} e^{-j 2 \pi m\left(\left(\left(\Delta f\left(r_{t}+r_{r}\right)\right) / c\right)-\left(\left(d_{t} \sin \theta_{t}\right) / \lambda\right)\right)} \alpha_{n m}+n_{n}
$$

where $\lambda$ is the wavelength of the carrier and $n_{n} \sim \mathrm{CN}\left(0, \sigma_{n}^{2}\right)$.

For an array configuration of collocated transmit-receive, denoted by FDA-C, the parameters are set as $r_{t}=r_{r}=r, \theta_{t}=\theta_{r}=\theta$, and $d_{t}=d_{r}=d . \alpha_{n m}$ of all channels are fully correlated and can be denoted by a variable $\alpha$. Considering the ultimate detection performance, the weight $w_{m}$ is set as $w_{m}=e^{j 2 \pi m((2 r \Delta f / c)-(d \sin \theta / \lambda))}$. Therefore, the outputs of all receive channels can form a signal vector and be expressed as

$$
\mathbf{r}=\sqrt{\mathrm{ME}} \alpha e^{-j(4 \pi r / \lambda)} \mathbf{b}(\theta)+\mathbf{n},
$$

where $\quad \mathbf{r}=\left[r_{0}, r_{1}, \ldots, r_{N-1}\right]^{T}, \quad \mathbf{b}(\theta)=\left[b_{0}, b_{1}, \ldots, b_{N-1}\right]^{T}$, $b_{n}=e^{j 2 \pi n d \sin \theta / \lambda}, \mathbf{n}=\left[n_{0}, n_{1}, \ldots, n_{N-1}\right]^{T}, \mathbf{n} \sim \mathrm{CN}\left(0, \sigma_{n}^{2} \mathbf{I}_{N}\right)$, and $\mathbf{I}_{N}$ is an $N$ identity matrix.

For a configuration of collocated transmit distributed receive, denoted by FDA-D, the reflection coefficients of different receive channels are uncorrelated. However, they are fully correlated for all transmit channels. Therefore, the reflection coefficient $\alpha_{n m}$ is represented by a variable $\alpha_{n}$. Considering the ultimate performance, the weight $w_{m}$ is set as $w_{m}=e^{j 2 \pi m\left(\Delta f\left(r_{t}+r_{r}\right) / c-d_{t} \sin \theta_{t} / \lambda\right)}$. Therefore, the outputs of all receive channels can form a vector that is written as

$$
\mathbf{r}=\sqrt{\mathrm{ME}} e^{-j 2 \pi\left(\left(r_{t}+r_{r}\right) / \lambda\right)} \mathbf{b}\left(\theta_{r}\right) \circ \boldsymbol{a}+\mathbf{n},
$$

where $\circ$ is the Hadamard product, $\alpha=\left[\alpha_{0}, \alpha_{1}, \ldots, \alpha_{(N-1)}\right]^{T}$, $\alpha \sim \mathrm{CN}\left(0, \mathbf{I}_{N}\right)$, and $\mathbf{n} \sim \mathrm{CN}\left(0, \sigma_{n}^{2} \mathbf{I}_{N}\right)$.

2.2. Signal Model of FDA-MIMO. The schematic diagram of signal processing at the $n$th receive channel is shown in Figure 1(b). After firstly amplified by a LNA, the received signal in (4) is mixed with $e^{-j 2 \pi f_{0} t}$ and then fed into a bank of $M$-matched filters $h_{m}(t)=\phi_{m}^{*}(-t) e^{j 2 \pi m \Delta f t}$ to separate the transmit signals. The separated signal is mixed with $s_{m f}(t)=e^{-j 2 \pi m \Delta f t}$, and the output can be expressed as

$$
\begin{aligned}
r_{n m}(t)= & {\left[\int_{n} r_{n}(\tau) h_{m}(t-\tau) \mathrm{d} \tau\right] s_{m f}^{*}(t) } \\
= & \sqrt{\frac{E}{M}} \sum_{l=0}^{M-1}\left(w_{l} \alpha_{n l}\left(\int_{T} \phi_{l}\left(\tau-\tau_{n l}\right) \phi_{m}^{*}(\tau-t) e^{j 2 \pi(l-m) \Delta f \tau} \mathrm{d} \tau\right) e^{-j 2 \pi f_{l} \tau_{n l}}\right) \\
& +\left(\int_{T} n_{n}(\tau) \phi_{m}^{*}(\tau-t) e^{j 2 \pi m \Delta f(t-\tau)} \mathrm{d} \tau\right) e^{-j 2 \pi m \Delta f t} .
\end{aligned}
$$

According to the orthogonal properties in (2) and (3), let $l=m$ and $t=\tau_{n m}$, and then substitute (5) into (9); we can obtain

$$
r_{n m}=\sqrt{\frac{E}{M}} w_{m} \alpha_{n m} e^{-j 2 \pi f_{0}\left(r_{t}+r_{r} / c\right)} e^{-j 2 \pi m\left(\left(\Delta f\left(r_{t}+r_{r}\right) / c\right)-\left(f_{0} d_{t} \sin \theta_{t} / c\right)\right)} e^{j 2 \pi f_{0}\left(n d_{r} \sin \theta_{r} / c\right)}+n_{n m}
$$

where $n_{n m} \sim \mathrm{CN}\left(0, \sigma_{n}^{2}\right)$.

For a configuration of collocated transmit-receive, denoted by FDA-MIMO-C, the parameter setting is the same as that of the standard FDA. Equation (10) can be rewritten as

$$
r_{n m}=\sqrt{\frac{E}{M}} \alpha e^{-j(4 \pi r / \lambda)} e^{j 2 \pi f_{0}(n d \sin \theta / c)}+n_{n m}
$$

Therefore, the final output in Figure 1(b) is the sum of all $r_{n m}$ and can be expressed as

$$
\begin{aligned}
r_{n} & =\sum_{m=0}^{M-1} r_{n m}=M \sqrt{\frac{E}{M}} \alpha e^{-j(4 \pi r / \lambda)} e^{j 2 \pi f_{0}(n d \sin \theta / c)}+\sum_{m=0}^{M-1} n_{n m} \\
& =\sqrt{M E} \alpha e^{-j(4 \pi r / \lambda)} e^{j 2 \pi f_{0}(n d \sin \theta / c)}+n_{n},
\end{aligned}
$$


where $n_{n}$ is the linear combination of $n_{n m}$ that obeys a complex Gaussian distribution; therefore, $n_{n} \sim \mathrm{CN}\left(0, M \sigma_{n}^{2}\right)$.

The signal vector composed of all $r_{n}$ can be written as

$$
\mathbf{r}=\sqrt{M E} \alpha e^{-j(4 \pi r / \lambda)} \mathbf{b}(\theta)+\mathbf{n},
$$

where $\mathbf{n} \sim \mathrm{CN}\left(0, M \sigma_{n}^{2} \mathbf{I}_{N}\right)$

For a configuration of collocated transmit distributed receive, denoted by FDA-MIMO-D, the parameter setting is also the same as that of the standard FDA. Therefore, the final output $r_{n}$ in Figure 1(b)) and the signal vector formed by $r_{n}$ can be expressed as, respectively,

$$
r_{n}=\sum_{m=0}^{M-1} r_{n m}=\sqrt{M E} e^{-j 2 \pi\left(r_{t}+r_{r} / \lambda\right)} \alpha_{n} e^{j 2 \pi f_{0}\left[n d_{r} \sin \theta_{r} / c\right]}+n_{n}
$$

$$
\mathbf{r}=\sqrt{M E} e^{-j 2 \pi\left(r_{t}+r_{r} / \lambda\right)} \mathbf{b}\left(\theta_{r}\right) \circ \boldsymbol{\alpha}+\mathbf{n},
$$

where $\mathbf{n} \sim \mathrm{CN}\left(0, M \sigma_{n}^{2} \mathbf{I}_{M}\right)$.

2.3. The Analysis of Noise Responses of the Bandpass Filter and Matched Filter. In the actual system, as long as the frequency range of the uniform distribution of the noise power spectrum is much larger than the working frequency band of a system, it can be regarded as a white noise. Supposing that the radar is a system with a bandwidth of $B$, the power spectrum density function of the noise is $P(f)=\sigma_{n}^{2}, f_{c}-B / 2<f<f_{c}+B / 2$, and the corresponding correlation function is $R(\tau)=B \sigma_{n}^{2} e^{-j 2 \pi f_{c} \tau} \operatorname{sinc}(\pi B \tau)$, where $B \sigma_{n}^{2}$ is the noise variance, also known as average power. In Figure 1(a), the bandwidth of each subband filter is equal to $B / M$, and its correlation function is $R(\tau)=(B / M) \sigma_{n}^{2} e^{-j 2 \pi f_{c} \tau} \operatorname{sinc}(\pi B \tau)$, where $(B / M) \sigma_{n}^{2}$ is the noise variance. It can be seen that the variance of the input noise of the bandpass filter bank is $M$ times that of the output noise of the $m$ th subband filter. Here, the input noise term $n_{n}(t)$ in Figure $1(\mathrm{a})$ is assumed to be a white, zero-mean, complex Gauss random process. Its mean and variance are zero and $\sigma_{n}^{2}$, respectively. The output of the $m$ th subband filter, denoted by $n_{n m}$, is a narrowband Gaussian noise. Therefore, the mean and variance of $n_{n m}$ are zero and $(1 / M) \sigma_{n}^{2}$, respectively. The final output noise $n_{n}$ in (6) is the sum of all $n_{n m}$, as follows:

$$
n_{n}=\sum_{m=0}^{M-1} n_{n m}
$$

Therefore, the noise term $n_{n}$ in (6) obeys a complex Gauss distribution $n_{n} \sim \mathrm{CN}\left(0, \sigma_{n}^{2}\right)$.

For the matched filter in Figure 1(b), it can be seen from (9) and (10) that

$$
n_{n m}=\left(\int_{T} n_{n}(\tau) \phi_{m}^{*}(\tau-t) e^{j 2 \pi m \Delta f(t-\tau)} \mathrm{d} \tau\right) e^{-j 2 \pi m \Delta f t}
$$

The mean and variance of $n_{n m}$ are as follows:

$$
\begin{aligned}
E\left\{n_{n m}\right\} & =E\left\{\left(\int_{T} n_{n}(\tau) \phi_{m}^{*}(\tau-t) e^{j 2 \pi m \Delta f(t-\tau)} \mathrm{d} \tau\right) e^{-j 2 \pi m \Delta f t}\right\} \\
& =\left(\int_{T} E\left\{n_{n}(\tau)\right\} \phi_{m}^{*}(\tau-t) e^{j 2 \pi m \Delta f(t-\tau)} \mathrm{d} \tau\right) e^{-j 2 \pi m \Delta f t}=0, \\
\operatorname{var}\left\{n_{n m}\right\} & =E\left\{\iint_{T} n_{T}(\tau) n_{n}^{*}(u) \phi_{m}^{*}(\tau-t) \phi_{m}(u-t) e^{j 2 \pi m \Delta f(u-\tau)} \mathrm{d} \tau \mathrm{d} u\right\} \\
& =\iint_{T} E\left\{n_{n}(\tau) n_{n}^{*}(u)\right\} \phi_{m}^{*}(\tau-t) \phi_{m}(u-t) e^{j 2 \pi m \Delta f(u-\tau)} \mathrm{d} \tau \mathrm{d} u \\
& =\sigma_{n}^{2} \int_{T} \phi_{m}^{*}(v-t) \phi_{m}(v-t) \mathrm{d} v=\sigma_{n}^{2} .
\end{aligned}
$$

Therefore, the noise term of the matched filter output is still a complex Gaussian distribution, i.e., $n_{n m} \sim \mathrm{CN}\left(0, \sigma_{n}^{2}\right)$. The final noise term $n_{n}$ in (12) obeys a complex Gauss distribution $n_{n} \sim \mathrm{CN}\left(0, M \sigma_{n}^{2}\right)$.

\section{Test Statistic and Its Probability Distribution}

Since we are concerned about the limit detection performance of various radar systems, the target distance and azimuth parameters are known, and the covariance of noise is also known in advance. In the Neyman-Pearson sense, the optimal detector is the likelihood ratio test (LRT) given by $[12,19$

$$
T=\ln \left(\frac{f\left(\mathbf{r} \mid H_{1}\right)}{f\left(\mathbf{r} \mid H_{0}\right)}\right) \gtrless_{H_{1}}^{H_{0}} \delta,
$$

where $f\left(\mathbf{r} \mid H_{1}\right)$ and $f\left(\mathbf{r} \mid H_{0}\right)$ are the probability density functions (PDFs) of the observation vector $\mathbf{r}$ with and 
without the signal, respectively. The threshold $\delta$ is determined by the desired probability of false alarm (PFA). $H_{1}$ denotes the target exists, and $H_{0}$ denotes the target does not exist.

Now, we further process the output signal of the $n$th receive channel. As shown in Figure 2, the detail of channel $n$ has been described in Figure 1(a) for the standard FDA and Figure 1(b) for FDA-MIMO, respectively. The new output is defined as $y_{n}=b_{n}^{*} r_{n}$ for the two radar systems. All of them form a new signal vector $\mathbf{y}=\left[y_{0}, y_{1}, \ldots, y_{N-1}\right]^{T}$. Therefore, we can get digital beamforming:

$$
y=\mathbf{b}(\theta)^{H} \mathbf{r} .
$$

3.1. Standard FDA. For FDA-C, the PDFs under both alternative and null hypotheses are as follows [12]:

$$
\begin{aligned}
f\left(\mathbf{r} \mid H_{1}\right) & =\int f\left(\mathbf{r} \mid H_{1}, \alpha\right) f(\alpha) \mathrm{d} \alpha \\
& =\frac{1}{\pi \pi^{N}\left|\sigma_{n}^{2}\right|^{N}} k e^{-\left(1 / \sigma_{n}^{2}\right)|r|^{2}} e^{\left(M E / \sigma_{n}^{2}\left(N M E+\sigma_{n}^{2}\right)\right)|y|^{2}},
\end{aligned}
$$

where $k$ is a constant term.

$$
f\left(\mathbf{r} \mid H_{0}\right)=\frac{1}{\pi^{N}\left|\sigma_{n}^{2}\right|^{N}} e^{-\left(1 / \sigma_{n}^{2}\right)\|\mathbf{r}\|^{2}} .
$$

Substituting (22) and (23) into (20), the test statistic can be obtained as follows:

$$
T_{F D A-C}=|y|^{2} \gtrless_{H_{1}}^{H_{0}} \delta,
$$

where $\delta$ is the updated threshold.

According to (7) and (21), we can get

$$
y=\mathbf{b}(\theta)^{H} \mathbf{r}=\left\{\begin{array}{l}
n H_{0} \\
\sqrt{M E} N e^{-j(4 \pi r / \lambda)} \alpha+n H_{1}
\end{array},\right.
$$

where $\alpha \sim \mathrm{CN}(0,1)$ and $n \sim \mathrm{CN}\left(0, M \sigma_{n}^{2}\right)$.

Therefore, the probability distribution of the test statistic $|y|^{2}$ is as follows:

$$
T_{F D A-C}=|y|^{2} \sim\left\{\begin{array}{l}
\frac{N \sigma_{n}^{2}}{2} \chi_{(2)}^{2} H_{0} \\
\left(\frac{N^{2} M^{2} E}{2 M}+\frac{N \sigma_{n}^{2}}{2}\right) \chi_{(2)}^{2} H_{1}
\end{array},\right.
$$

where $\chi_{(L)}^{2}$ denotes a chi-square random variable with $L$ degrees of freedom.

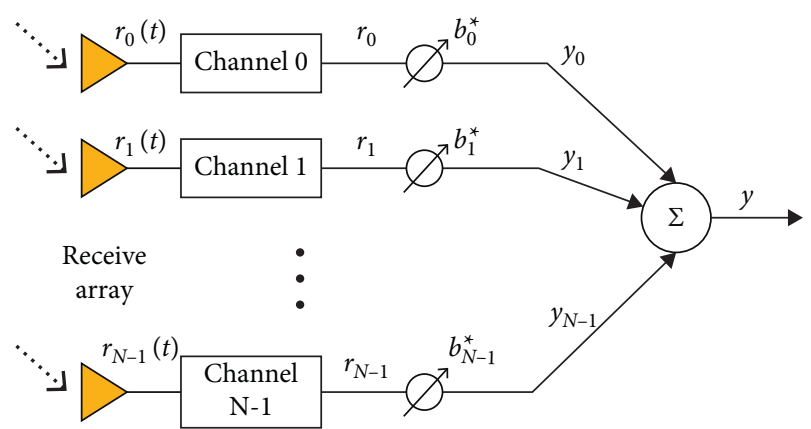

FIgURE 2: Digital beamforming at the receiving end for the standard FDA and FDA-MIMO radar.

For FDA-D, the PDFs under both alternative and null hypotheses are as follows:

$$
\begin{aligned}
f\left(\mathbf{r} \mid H_{1}\right) & =\int f\left(\mathbf{r}(t) \mid H_{1}, \boldsymbol{\alpha}\right) f(\mathbf{\alpha}) \mathrm{d} \mathbf{\alpha} \\
& =\frac{1}{\pi^{2 N}\left|\sigma_{n}^{2}\right|^{N}} k e^{-\left(1 / \sigma_{n}^{2}\right)\|\mathbf{r}\|^{2}} e^{\left(M E / \sigma_{n}^{2}\left(M E+\sigma_{n}^{2}\right)\right)\|\mathbf{y}\|^{2}},
\end{aligned}
$$

$$
f\left(\mathbf{r} \mid H_{0}\right)=\frac{1}{\pi^{N}\left|\sigma_{n}^{2}\right|^{N}} e^{-\left(1 / \sigma_{n}^{2}\right)\|\mathbf{r}\|^{2}}
$$

Substituting (27) and (28) into (20), we can get

$$
T_{F D A-D}=\|\mathbf{y}\|^{2} \gtrless_{H_{1}}^{H_{0}} \delta,
$$

where $\delta$ is the updated threshold.

According to (8), we can get

$$
\mathbf{y}=\mathbf{b}(\theta)^{H} \circ \mathbf{r}=\left\{\begin{array}{l}
\mathbf{n} H_{0} \\
\sqrt{M E} e^{-j 2 \pi\left(r_{t}+r_{r} / \lambda\right)} \mathbf{a}+\mathbf{n} H_{1}
\end{array},\right.
$$

where $\alpha \sim \mathrm{CN}\left(0, \mathbf{I}_{M}\right)$ and $\mathbf{n} \sim \mathrm{CN}\left(0, \sigma_{n}^{2} \mathbf{I}_{M}\right)$.

Therefore, the probability distribution of the test statistic $\|\mathbf{y}\|^{2}$ is as follows:

$$
T_{F D A-D}=\|\mathbf{y}\|^{2} \sim\left\{\begin{array}{l}
\frac{\sigma_{n}^{2}}{2} \chi_{(2 N)}^{2} H_{0} \\
\left(\frac{M E}{2}+\frac{\sigma_{n}^{2}}{2}\right) \chi_{(2 N)}^{2} H_{1}
\end{array}\right.
$$

3.2. FDA-MIMO. For FDA-MIMO-C, the PDFs under both alternative and null hypotheses are as follows [12]: 


$$
\begin{aligned}
f\left(\mathbf{r} \mid H_{1}\right) & =\int f\left(\mathbf{r} \mid H_{1}, \alpha\right) f(\alpha) \mathrm{d} \alpha \\
& =\frac{1}{\pi \pi^{N}\left|M \sigma_{n}^{2}\right|^{N}} k e^{-\left(1 / M \sigma_{n}^{2}\right)\|\mathbf{r}\|^{2}} e^{\left(1 / \sigma_{n}^{2}\right)\left(E / M N E+M \sigma_{n}^{2}\right)|y|^{2}},
\end{aligned}
$$

$$
f\left(\mathbf{r} \mid H_{0}\right)=\frac{1}{\pi^{N}\left|M \sigma_{n}^{2}\right|^{N}} e^{-\left(1 / M \sigma_{n}^{2}\right)\|r\|^{2}} .
$$

Substituting (32) and (33) into (20), the test statistic can be obtained as follows:

$$
T_{F D A-M I M O-C}=|y|^{2} \gtrless_{H_{1}}^{H_{0}} \delta,
$$

where $\delta$ is the updated threshold.

According to (13), we can obtain that

$$
y=\mathbf{b}(\theta)^{H} \mathbf{r}=\left\{\begin{array}{l}
n H_{0} \\
\sqrt{M E} N e^{-j(4 \pi r / \lambda)} \alpha+n H_{1}
\end{array},\right.
$$

where $\alpha \sim \mathrm{CN}(0,1)$ and $n \sim \mathrm{CN}\left(0, M N \sigma_{n}^{2}\right)$.

Therefore, the probability distribution of the test statistic $|y|^{2}$ is as follows:

$$
T_{F D A-M I M O-C}=|y|^{2} \sim\left\{\begin{array}{l}
\frac{M N \sigma_{n}^{2}}{2} \chi_{(2)}^{2} H_{0} \\
\left(\frac{M N^{2} E+M N \sigma_{n}^{2}}{2}\right) \chi_{(2)}^{2} H_{1}
\end{array}\right.
$$

For FDA-MIMO-D, the PDFs under both alternative and null hypotheses are as follows:

$$
\begin{aligned}
f\left(\mathbf{r} \mid H_{1}\right) & =\int f\left(\mathbf{r} \mid H_{1}, \alpha\right) f(\alpha) \mathrm{d} \alpha \\
& =\frac{1}{\pi \pi^{N}\left|M \sigma_{n}^{2}\right|^{N}} e^{-\left(1 / M \sigma_{n}^{2}\right)\|\mathbf{r}\|^{2}} k e^{\left(1 / \sigma_{n}^{2}\right)\left(E / M E+M \sigma_{n}^{2}\right)\|\mathbf{y}\|^{2}} \\
f\left(\mathbf{r} \mid H_{0}\right) & =\frac{1}{\pi^{N}\left|M \sigma_{n}^{2}\right|^{N}} e^{-\left(1 / M \sigma_{n}^{2}\right)\|\mathbf{r}\|^{2}}
\end{aligned}
$$

Substituting (37) and (38) into (20), the test statistic can be obtained as follows:

$$
T_{\text {FDA-MIMO-D }}=\|\mathbf{y}\|^{2} \gtrless_{H_{1}}^{H_{0}} \delta .
$$

According to (15), we can get

$$
\mathbf{y}=\left\{\begin{array}{l}
\mathbf{n} H_{0} \\
\sqrt{M E} e^{-j 2 \pi\left(r_{t}+r_{r} / \lambda\right)} \boldsymbol{\alpha}+\mathbf{n} H_{1}
\end{array},\right.
$$

where $\mathbf{n} \sim \mathrm{CN}\left(0, M \sigma_{n}^{2} \mathbf{I}_{M}\right)$.

Therefore, the probability distribution of the test statistic $\|\mathbf{y}\|^{2}$ is as follows:

$$
T_{F D A-M I M O-D}=\|\mathbf{y}\|^{2} \sim\left\{\begin{array}{l}
\frac{M \sigma_{n}^{2}}{2} \chi_{(2 N)}^{2} H_{0} \\
\left(\frac{M E+M \sigma_{n}^{2}}{2}\right) \chi_{(2 N)}^{2} H_{1}
\end{array}\right.
$$

\section{Detection Performances of Various Radar Systems}

The solution method of target detection probability is the same for the radar systems mentioned above. The probability of false alarm $P_{f a}$ is known in advance, and we can obtain the threshold $\delta$ by solving $P_{f a}=P\left(T>\delta \mid H_{0}\right)$. The detection probability is then obtained by $P_{d}=P\left(T>\delta \mid H_{1}\right)$. Therefore, we only deduce the derivation process of the detection probability of the FDA-C radar, and the detection probability of the remaining radars is given directly.

With the aid of (26), the probability of false alarm can be expressed as

$$
P_{f a}=P\left(T>\delta \mid H_{0}\right)=P\left(\frac{N \sigma_{n}^{2}}{2} \chi_{(2)}^{2}>\delta\right)=P\left(\chi_{(2)}^{2}>\frac{2 \delta}{N \sigma_{n}^{2}}\right) .
$$

The detection threshold in (42) can be obtained as

$$
\delta=\frac{N \sigma_{n}^{2}}{2} F_{\chi_{(2)}^{2}}^{-1}\left(1-P_{f a}\right),
$$

where $F_{\chi_{L I}^{2}}^{-1}$ is the inverse cumulative distribution function of a chi-square random variable with $L$ degrees of freedom.

The probability of detection is given by

$$
\begin{aligned}
P_{d}^{(F D A-C)} & =P\left(T>\delta \mid H_{1}\right) \\
& =P\left(\left(\frac{N^{2} M^{2} E}{2 M}+\frac{N \sigma_{n}^{2}}{2}\right) \chi_{(2)}^{2}>\delta\right) \\
& =1-F_{\chi_{(2)}^{2}}\left(\frac{1}{1+M N \rho} F_{\chi_{(2)}^{2}}^{-1}\left(1-P_{f a}\right)\right),
\end{aligned}
$$

where $\rho=E / \sigma_{n}^{2}$ is defined as the SNR that is the ratio between the total transmitted energy and the noise level per receive element.

Then, the detection probability of the remaining radar system is given directly as follows:

$$
\begin{aligned}
P_{d}^{(F D A-D)} & =1-F_{\chi_{(2 N)}^{2}}\left(\frac{1}{1+M \rho} F_{\chi_{(2 N)}^{2}}^{-1}\left(1-P_{f a}\right)\right), \\
P_{d}^{(F D A-M I M O-C)} & =1-F_{\chi_{(2)}^{2}}\left(\frac{1}{1+N \rho} F_{\chi_{(2)}^{2}}^{-1}\left(1-P_{f a}\right)\right), \\
P_{d}^{(F D A-M I M O-D)} & =1-F_{\chi_{(2 N)}^{2}}\left(\frac{1}{1+\rho} F_{\chi_{(2 N)}^{2}}^{-1}\left(1-P_{f a}\right)\right) .
\end{aligned}
$$


Here, we present the detection probabilities of the phased-array (PHA) and MIMO radar for comparison, as follows [12]:

$$
\begin{aligned}
P_{d}^{(\mathrm{PHA})} & =1-F_{\chi_{(2)}^{2}}\left(\frac{1}{1+M N \rho} F_{(2)}^{-1}\left(1-P_{f a}\right)\right), \\
P_{d}^{(\mathrm{MIMO})} & =1-F_{(2 M N)}^{-1}\left(\frac{M}{M+\rho} F_{\chi_{(2 M N)}^{2}}^{-1}\left(1-P_{f a}\right)\right) .
\end{aligned}
$$

Besides the detection probability, we also employ another performance measure that is the detector's SNR (also referred to as divergence) [12]. It, denoted by $\beta$, is defined as follows:

$$
\beta=\frac{\left|E\left(T \mid H_{0}\right)-E\left(T \mid H_{1}\right)\right|^{2}}{(1 / 2)\left[\operatorname{Var}\left(T \mid H_{0}\right)+\operatorname{Var}\left(T \mid H_{1}\right)\right]} .
$$

Here, we also only deduce the derivation process of the detector's SNR of the FDA-C radar, and the detector's SNR of the remaining radars is given directly.

Based on (26), directly using $E\left(\chi_{(L)}^{2}\right)=L$ and $\operatorname{Var}\left(\chi_{(L)}^{2}\right)=2 L$, we can get

$$
\begin{gathered}
E\left(T_{F D A-C} \mid H_{0}\right)=N \sigma_{n}^{2}, \\
E\left(T_{F D A-C} \mid H_{1}\right)=N^{2} M E+N \sigma_{n}^{2}, \\
\operatorname{Var}\left(T_{F D A-C} \mid H_{0}\right)=N^{4}\left(\sigma_{n}^{2}\right)^{2}, \\
\operatorname{Var}\left(T_{F D A-C} \mid H_{1}\right)=\left(N^{2} M E+N \sigma_{n}^{2}\right)^{2} .
\end{gathered}
$$

Substituting (51)-(54) into (50), the detector's SNR can be obtained as follows:

$$
\beta^{(F D A-C)}=\frac{M^{2} N^{2} \rho^{2}}{1+M N \rho+M^{2} N^{2} \rho^{2} / 2} .
$$

The detector's SNR of the remaining radars is given directly as follows:

$$
\begin{aligned}
\beta^{(F D A-D)} & =\frac{{ }^{M} N^{2} \rho^{2}}{1+N \rho+N^{2} \rho^{2} / 2}, \\
\beta^{(F D A-M I M O-C)} & =\frac{N^{2} \rho^{2}}{1+N \rho+N^{2} \rho^{2} / 2}, \\
\beta^{(F D A-M I M O-D)} & =\frac{N \rho^{2}}{1+\rho+\rho^{2} / 2}, \\
\beta^{(M I M O)} & =\frac{\rho^{2} M}{N\left(1+\rho^{2} /\left(2 N^{2}\right)+\rho / N\right)} .
\end{aligned}
$$

\section{Numerical Simulations}

In this section, several numerical examples are presented to compare the performance of various radar systems, especially for the low SNR environment. From (44), (48), and (55), it can be seen that the PHA and FDA-C have the same performance. Therefore, we only use FDA-C for comparison in the following examples.

Figure 3 depicts the performance of the detector's SNR of various radar systems. The radar parameters are set as $M=5$ and $N=5$. The probability of false alarm is fixed at $P_{f a}=1 \times 10^{-5}$. The horizontal axis represents the signal-tonoise ratio $\rho$, and the vertical axis is the detector's SNR.

At high SNR, it can be seen that the MIMO radar has superior detection performance than that of others. High SNR ensures that each transceiver channel can detect target signals. Meanwhile, it sees a different aspect of the target and can exploit spatial diversity to overcome target fading. By comparison, FDA-C and FDA-MIMO-C radars have the worst performance. These two radars can realize a coherent processing gain, but they have no spatial diversity gain. When the reflection coefficient $\alpha$ in a special aspect of the target is small, they will suffer considerable performance degradation. For the other two FDA-D and FDA-MIMO-D, their performances are between $\mathrm{MIMO}$ and FDA-C and FDA-MIMO-C.

At low SNR, all channels of the MIMO radar cannot effectively detect the target signal, and spatial diversity gain cannot be used, resulting in that its performance is worse, whereas the performances of PHA and FDA-C are optimal due to their coherent gain ensuring effective detection for a small value of $\alpha$. The performance of others is between MIMO and PHA and FDA-C.

The detection probability of various radar systems is shown in Figure 4. It can be seen that FDA-C and FDA-D have a good performance at lower SNR. The performances of MIMO and FDA-MIMO-D improve rapidly with the increase of SNR. These numerical results are for $M=5$ and $N=5$. However, their performance will change with the number of array elements.

Generally, people pay much attention to the detection performance under the low SNR condition. Here, we continue to study how the number of array elements influences the detection performance of various radar systems in a low SNR environment. In Figures 5(a)-5(c), the number of receive elements changes from 1 to 100 , and the number of transmit elements is set as 2, 5, and 10 from Figures 5(a) to 5 (c), respectively. The probability of false alarm and SNR are set as $P_{f a}=1 \times 10^{-5}$ and $\rho=0 \mathrm{~dB}$, respectively. It can be seen that the detection probability will be improved with the increase of the number of receive elements for all radar systems mentioned above. Meanwhile, the performances of FDA-C and FDA-D are further improved with the increase of the number of transmit elements. However, the performance of FDA-MIMO-C and FDA-MIMO-D will not be improved with the increase of the number of transmit elements, and the performance of MIMO has even showed the trend of deterioration. We will try to explain this phenomenon below. When the total power of the radar is constant, less power is radiated by each transmitting channel due to the more transmitting array elements. This results in a reduction in the signal-to-noise ratio of the signal received by the virtual channel, which in turn degrades the detection 


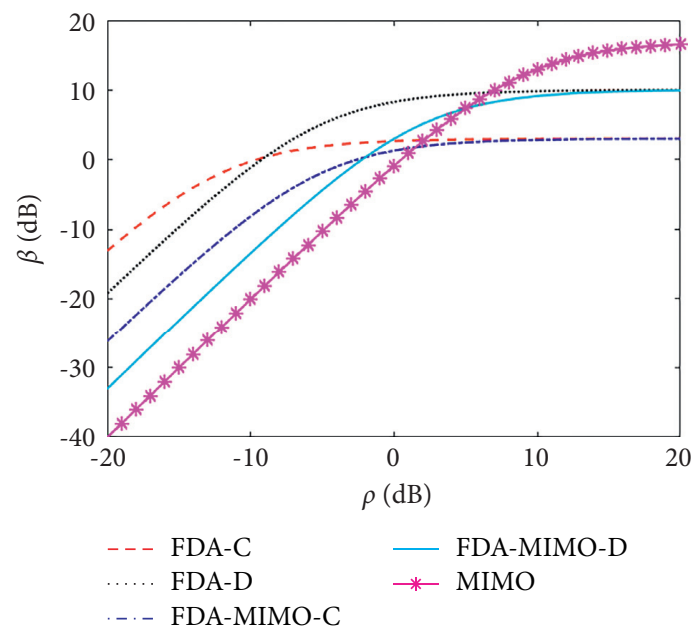

FIgURE 3: Detector's SNR of various systems. $M=N=5$.

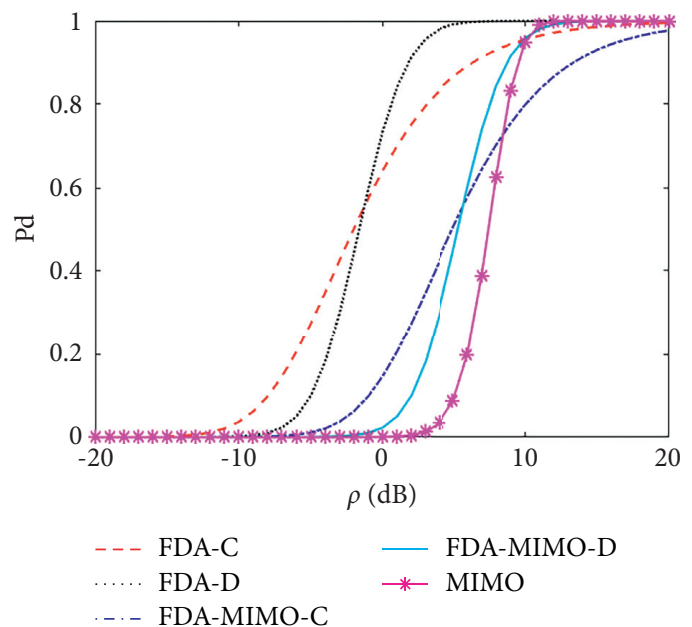

Figure 4: Illustration of target detection probability of various radar systems.

performance. About the above problems, longer transmission/integration times will be needed to improve the detection performance.

Figure 6 shows the simulation results with the SNR of $10 \mathrm{~dB}, 0 \mathrm{~dB},-10 \mathrm{~dB}$, and $-20 \mathrm{~dB}$, where the value range of the horizontal axis is adjusted adaptively according to the curve. The number of transmit elements in the figure is set to be 2 . It can be seen that the configuration of distributed arrays, i.e., FDA-D, FDA-MIMO-D, and MIMO, can use fewer elements to rapidly achieve the best performance for $\rho=10 \mathrm{~dB}$, whereas the configuration of collocated arrays, i.e., FDA-C and FDA-MIMO-C, has suboptimal performance. From Figures 6(b)-6(d), we can see that more elements are needed to ensure the detection performance as the SNR continues to decline. Especially at lower $\operatorname{SNR} \rho=-20 \mathrm{~dB}$, the performance of collocated configurations, i.e., FDA-C and FDA-MIMO-C, is relatively optimal, whereas the distributed configurations, i.e., FDA-D, FDA-MIMO-D, and MIMO, are the worst.

Therefore, when detecting low RCS targets, to use the advantages of the distributed radar effectively, i.e., FDA-D, FDA-MIMO-D, and MIMO, it is necessary to ensure that each transceiver channel or at least most of the transceiver channels can detect the effective target signal, that is, to ensure sufficient signal-to-noise ratio of most of the transceiver channels. 

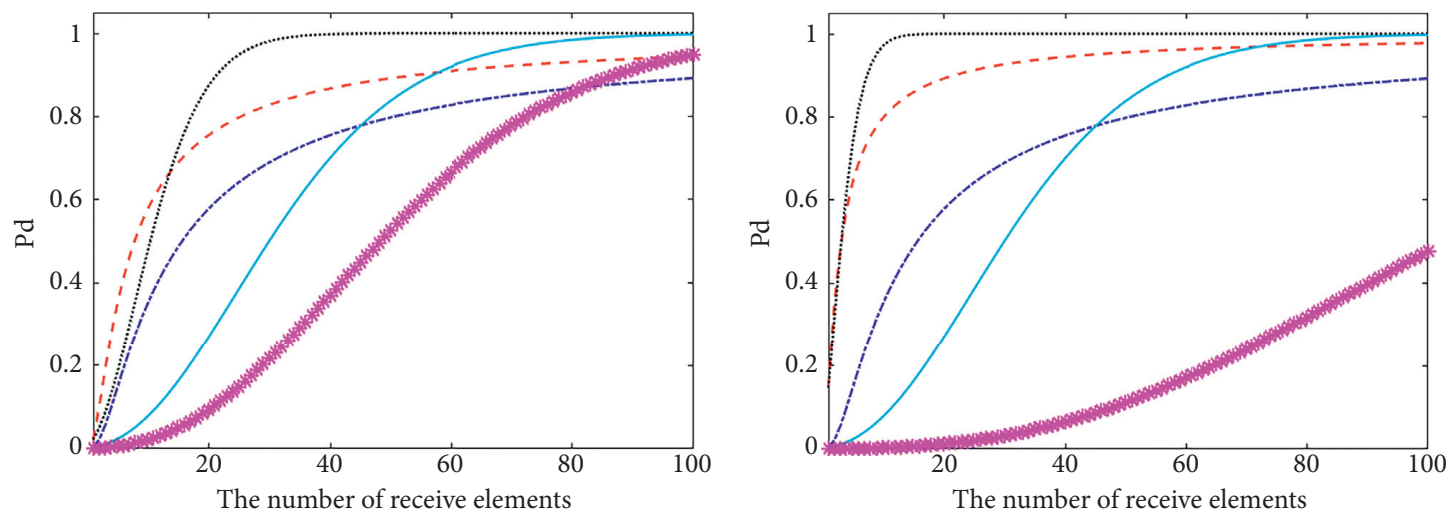

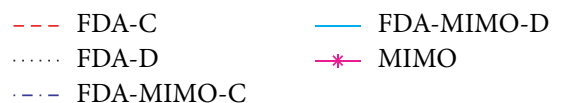

(a)

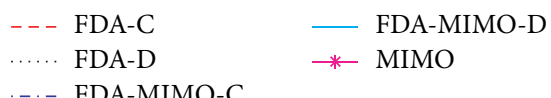

... FDA-MIMO-C

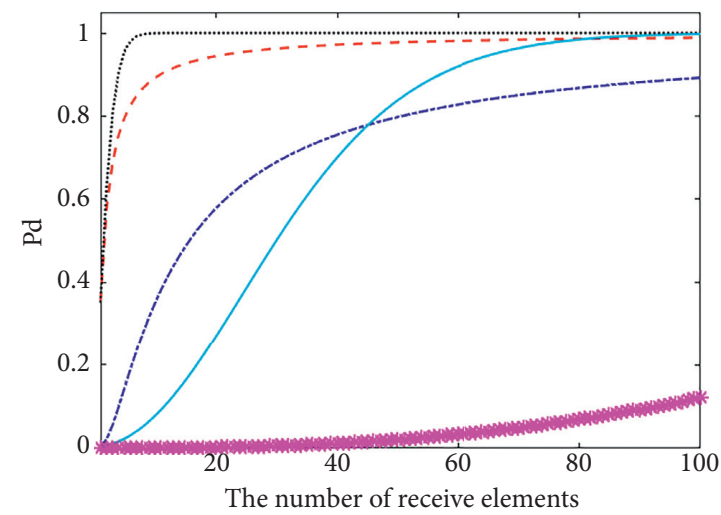

(b)

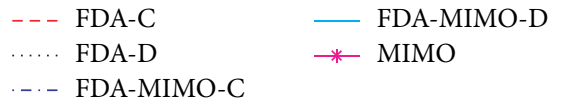

(c)

FIGURE 5: The influence of the array element number on detection probability at the SNR of $0 \mathrm{~dB}$. (a) $M=2$. (b) $M=5$. (c) $M=10$.

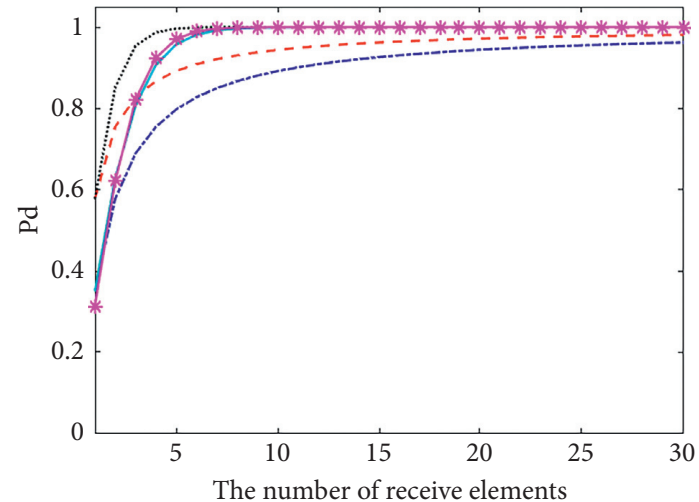

- - FDA-C

FDA-D

... FDA-MIMO-C
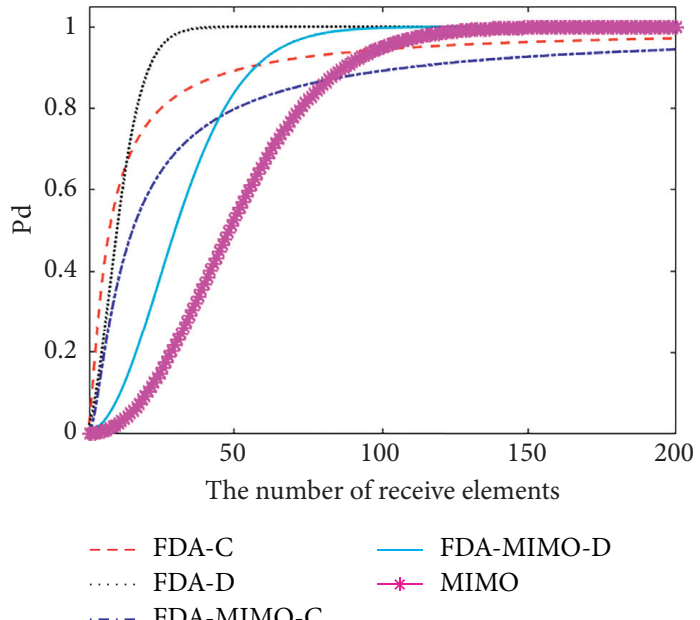

(b)

(a)

FIgURE 6: Continued. 


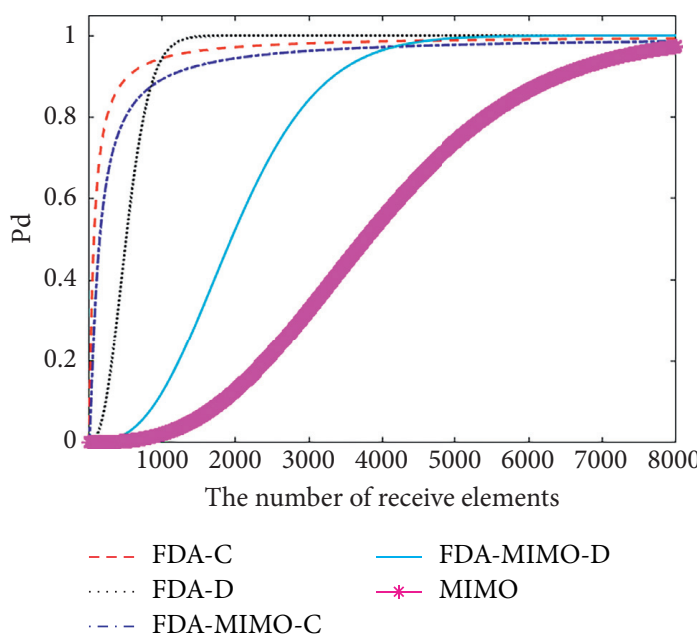

(c)

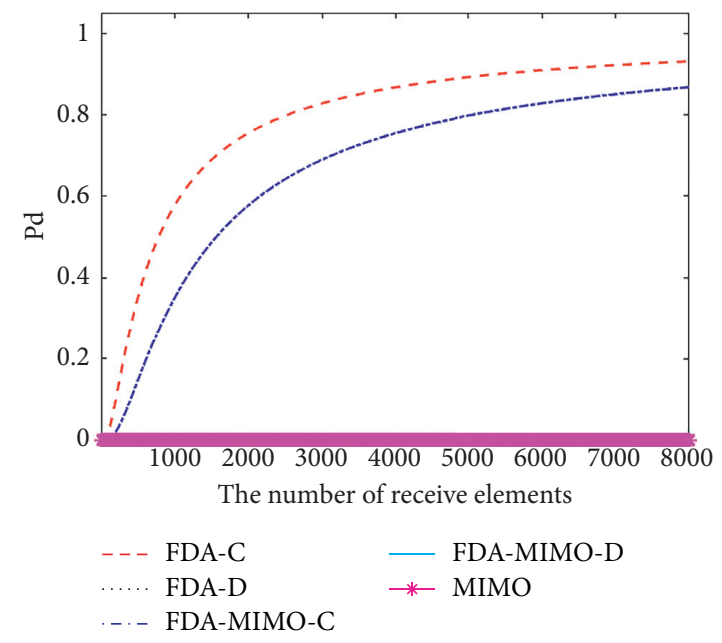

(d)

Figure 6: Detection probability of various radar systems with the SNR of $10 \mathrm{~dB}, 0 \mathrm{~dB},-10 \mathrm{~dB}$, and $-20 \mathrm{~dB}$. (a) $\rho=10 \mathrm{~dB}$. (b) $\rho=0 \mathrm{~dB}$. (c) $\rho=-10 \mathrm{~dB}$. (d) $\rho=-20 \mathrm{~dB}$.

\section{Conclusions}

In this paper, the target detection performance of several radars with different array configurations is comparatively analyzed in Gaussian clutter and noise. We mainly focus on the design of the receiver structure of FDA and FDA-MIMO radars and their array signal processing scheme. The likelihood ratio test statistics and the test statistic distributions are derived in the Neyman-Pearson sense for the two array configurations, namely, collocated transmit-receive and collocated transmit distributed receive. The numerical results show that the detection probability of FDA-C and FDA-D will be improved with the increase of the number of transmit and receive elements, whereas the detection probability of FDA-MIMO-C and FDA-MIMO-D can improve their performances only by increasing the number of receive elements. For the configuration of distributed arrays, i.e., FDA-D, FDA-MIMO-D, and MIMO, it can use fewer elements to rapidly achieve the best performance at high SNR. As the SNR continues to decline, FDA-C and FDA-MIMO-C are relatively optimal, which needs more elements to ensure their detection performance. By comprehensive analysis, in the case of low SNR, FDA-C and FDA-MIMO-C are the best choice for target detection, while in the case of high SNR, FDA-D, FDA-MIMO-D, and MIMO can be the optimal choice. All conclusions of the paper are drawn under the condition of a single snapshot. For multiple snapshots using long-term accumulation, a conclusion different from this paper may be drawn, which is the subsequent focus of this paper.

\section{Data Availability}

The data used to support the findings of this study are included within the article.

\section{Conflicts of Interest}

The authors declare that they have no conflicts of interest.

\section{Acknowledgments}

This research was funded in part by the Natural Science Foundation of Hebei Province (Grant no. F2019210253).

\section{References}

[1] P. Antonik, M. C. Wicks, H. D. Griffiths, and C. J. Baker, "Frequency diverse array radars," in Proceedings of the IEEE Conference on Radar, pp. 215-217, Verona, NY, USA, April 2006.

[2] Y. Wang and S. Zhu, "Main-beam range deceptive jamming suppression with simulated annealing FDA-MIMO radar," IEEE Sensors Journal, vol. 20, no. 16, pp. 9056-9070, 2020.

[3] J. Xu, S. Zhu, and G. Liao, "Range ambiguous clutter suppression for airborne FDA-STAP radar," IEEE Journal of Selected Topics in Signal Processing, vol. 9, no. 8, pp. 16201631, 2015.

[4] Y. Huang, G. Liao, J. Xu, J. Li, and D. Yang, "GMTI and parameter estimation for MIMO SAR system via fast interferometry RPCA method," IEEE Transactions on Geoscience and Remote Sensing, vol. 56, no. 3, pp. 1774-1787, 2018.

[5] H. Shao, J. Dai, j. Xiong, H. Chen, and W.-Q. Wang, "Dotshaped range-angle beampattern synthesis for frequency diverse array," IEEE Antennas and Wireless Propagation Letters, vol. 15, pp. 1703-1706, 2016.

[6] T. Chen and D. Xia, "Lagrange programming neural network approach for frequency diverse array beampattern synthesis," Circuits, Systems, and Signal Processing, vol. 39, no. 1, pp. $439-455,2020$.

[7] M. Mahmood and H. Mir, "FDA Transmit beampattern synthesis using piecewise trigonometric frequency offset," IET Radar, Sonar \& Navigation, vol. 13, no. 7, pp. 1149-1153, 2019.

[8] Y. Liao, W.-Q. Wang, and Z. Zheng, "Frequency diverse array beampattern synthesis using symmetrical logarithmic frequency offsets for target indication," IEEE Transactions on Antennas and Propagation, vol. 67, no. 5, pp. 3505-3509, 2019.

[9] A.-M. Yao, w. Wu, and D.-G. Fang, "Frequency diverse array antenna using time-modulated optimized frequency offset to obtain time-invariant spatial fine focusing beampattern," 
IEEE Transactions on Antennas and Propagation, vol. 64, no. 10, pp. 4434-4446, 2016.

[10] J. Xu, G. Liao, S. Zhu, L. Huang, and H. C. So, "Joint range and angle estimation using MIMO radar with frequency diverse array," IEEE Transactions on Signal Processing, vol. 63, no. 13, pp. 3396-3410, 2015.

[11] J. Xu, G. Liao, S. Zhu, and H. C. So, "Deceptive jamming suppression with frequency diverse MIMO radar," Signal Processing, vol. 113, pp. 9-17, 2015.

[12] E. Fishler, A. Haimovich, R. S. Blum, L. J. Cimini, D. Chizhik, and R. A. Valenzuela, "Spatial diversity in radars-models and detection performance," IEEE Transactions on Signal Processing, vol. 54, no. 3, pp. 823-838, 2006.

[13] J. Xiong, W.-Q. Wang, and K. Gao, "FDA-MIMO radar range-angle estimation: CRLB, MSE, and resolution analysis," IEEE Transactions on Aerospace and Electronic Systems, vol. 54, no. 1, pp. 284-294, 2018.

[14] J. Xu, G. Liao, Y. Zhang, H. Ji, and L. Huang, "An adaptive range-angle-Doppler processing approach for FDA-MIMO radar using three-dimensional localization," IEEE Journal of Selected Topics in Signal Processing, vol. 11, no. 2, pp. 309-320, 2017.

[15] C. Cui, J. Xu, R. Gui, W.-Q. Wang, and W. Wu, "Search-free DOD, DOA and range estimation for bistatic FDA-MIMO radar," IEEE Access, vol. 6, pp. 15431-15445, 2018.

[16] L. Lan, A. Marino, A. Aubry et al., "GLRT-based adaptive target detection in FDA-MIMO radar," IEEE Transactions on Aerospace and Electronic Systems, vol. 57, no. 1, pp. 597-613, 2021.

[17] L. Lan, M. Rosamilia, A. Aubry, A. Demaio, and G. Liao, "Single-snapshot angle and incremental range estimation for FDA-MIMO radar," IEEE Transactions on Aerospace and Electronic Systems, vol. 99, p. 1, 2021.

[18] Q. Liu, J. Xu, Z. Ding, and H. C. So, "Target localization with jammer removal using frequency diverse array," IEEE Transactions on Vehicular Technology, vol. 69, no. 10, pp. 11685-11696, 2020.

[19] Y. Zhu, L. Liu, Z. Lu, and S. Zhang, "Target detection performance analysis of FDA-MIMO radar," IEEE Access, vol. 7, pp. 164276-164285, 2019.

[20] Y. Xu and K.-M. Luk, "Enhanced transmit-receive beamforming for frequency diverse array," IEEE Transactions on Antennas and Propagation, vol. 68, no. 7, pp. 5344-5352, 2020 . 